\title{
Change in prevalence and molecular characteristics of isoniazid-resistant tuberculosis over a 10-year period in China
}

Fengmin Huo ${ }^{1 \dagger}$, Jie Lu ${ }^{2 \dagger}$, Zhaojing Zong ${ }^{1}$, Wei Jing ${ }^{1}$, Jin Shi ${ }^{2}$, Yifeng $\mathrm{Ma}^{1}$, Lingling Dong ${ }^{1}$, Liping Zhao ${ }^{1}$, Yufeng Wang ${ }^{1}$, Hairong Huang ${ }^{1}$ and Yu Pang ${ }^{1 *}$

\begin{abstract}
Background: Isoniazid (INH) represents the cornerstone for the treatment of cases infected with Mycobacterium tuberculosis (MTB) strains. Several molecular mechanisms have been shown to be the major causes for INH resistance, while the dynamic change of mutations conferring INH resistance among MTB strains during the past decade is still unknown in China.

Methods: In this study, we carried out a comparative analysis of the INH minimal inhibitory concentration (MIC) distribution, and investigate the dynamic change of molecular characteristics among INH-resistant MTB strains between 2005 and 2015.

Results: The proportion of INH resistance $(39.0 \%, 105 / 269)$ in 2015 was significantly higher than in 2005 (30.0\%, 82/273; $P=0.03)$. Among 269 isolates collected in 2015, 76 (28.3\%, 76/269) exhibited high-level INH-resistance (MIC $\geq 32 \mathrm{mg} / \mathrm{L})$, which was significantly higher than that in $2005(20.5 \%, 56 / 273, P=0.04)$. In addition, a significantly higher percentage of INH-resistant isolates carried inhA promoter mutations in 2015 (26.7\%) versus that in 2005 (14.6\%, $P=0.04)$, while no significant difference was observed in the rates of isolates containing katG mutations between 2005 (76.8\%) and 2015 (70.5\%, $P=0.33)$. Notably, the proportion of MTB isolates with inhA mutations $(26.7 \%, 28 / 105)$ for patients who had previous exposure to protionamide (PTH) was higher than that for patients who had no previous exposure to PTH $(21.4 \%, 6 / 28)$.

Conclusions: In conclusion, our results demonstrated that the proportion of INH-resistant MTB isolates significantly increased during the last decade, which was mainly attributed to an increase of high-level INH-resistant MTB. In addition, prior exposure to PTH may be associated with the increased frequency of INH-resistant tuberculosis strains with inhA mutations in China.
\end{abstract}

Keywords: Tuberculosis, Isoniazid, Drug resistance, inhA, Protionamide

\section{Background}

Tuberculosis (TB), is a serious global public health concern, with an incidence of 10.0 million new cases and 1.6 million deaths in 2017 [1]. Despite achieving great progress in curbing the TB epidemic during the last two decades, the efforts to control TB are threatened by the

\footnotetext{
* Correspondence: pangyupound@163.com

${ }^{\dagger}$ Fengmin Huo and Jie Lu contributed equally to this work.

${ }^{1}$ National Clinical Laboratory on Tuberculosis, Beijing Key laboratory on

Drug-resistant Tuberculosis Research, Beijing Chest Hospital, Capital Medical

University, Beijing Tuberculosis and Thoracic Tumor Institute, No. 97,

Machang, Tongzhou District, Beijing 101149, China

Full list of author information is available at the end of the article
}

emergence of drug-resistant TB [2], especially multidrugresistant TB (MDR-TB), defined as the strains resistant to at least isoniazid (INH) and rifampicin (RIF) [3, 4]. As one of the most potent anti-TB drug, isoniazid, together with rifampicin, represents the cornerstone for the treatment of cases infected with RIF-susceptible TB strains [5]. Prior clinical trials have shown that the TB cases with initial INH resistance is at high risk for poor clinical outcomes among the cases receiving the standard first-line therapy regimen, who are more likely to progress to MDR-TB [6, 7]. Hence, the early detection of isoniazid resistance is essential to allow clinicians to adjust

(c) The Author(s). 2019 Open Access This article is distributed under the terms of the Creative Commons Attribution 4.0 International License (http://creativecommons.org/licenses/by/4.0/), which permits unrestricted use, distribution, and reproduction in any medium, provided you give appropriate credit to the original author(s) and the source, provide a link to the Creative Commons license, and indicate if changes were made. The Creative Commons Public Domain Dedication waiver (http://creativecommons.org/publicdomain/zero/1.0/) applies to the data made available in this article, unless otherwise stated. 
initial drug therapy, thereby bringing benefits for patients with INH-resistant TB.

Accurate molecular diagnosis of drug-resistant TB relies on the knowledge of the mechanisms conferring resistance to anti-TB drug [8]. Resistance to isoniazid is a complex process, and several genes, including katG, $\operatorname{inh} A, \operatorname{ahp} C$, kasA and $n d h$, involve in resistance to INH in MTB [9-11]. Of these targets, two molecular mechanisms have been shown to be the major causes for INH resistance: mutations in kat $G$ and mutations in the promoter region of inhA [11]. The katG gene encodes catalase/peroxidase enzyme in MTB, which is responsible for activating the pro-drug isoniazid [9]. As a consequence, the mutations in katG lead to the occurrence of INH resistance by the partial or total loss of catalase/ peroxidase activity. Multiple studies have shown that kat $G$ mutations are the most frequent genetic substitutions conferring INH resistance, especially in high-level INH resistant strains [12]. In the latter situation, mutations in the promoter region of $i n h A$ gene stimulate the overexpression of InhA, the target protein of INH, and cause INH resistance, which are usually associated with low-level resistance [12]. Although approximately $85 \%$ of all clinical INH-resistant isolates have the mutations in the two genes, the contribution of these two major mechanisms in INH resistance differ from one geographic region to another, indicating the genetic diversity of INH-resistant MTB strains circulating in various regions $[5,13,14]$.

China has been classified as global "hotspot" of drugresistant TB because of the high prevalence of drug-resistant TB [15]. Although several molecular epidemiological studies have been conducted to describe the molecular characteristics of INH-resistant strains from different regions of China [16-18], the dynamic change of mutations conferring INH resistance among MTB strains during the past decade is still unknown in this country. In a recent study, we identified the increased prevalence of INH resistance among MTB strains between 2005 and 2015 in China [19]. On the basis of our previous findings, we carried out a comparative analysis of the INH minimal inhibitory concentration (MIC) distribution, and investigate the dynamic change of molecular characteristics among INH-resistant MTB strains between 2005 and 2015 .

\section{Methods}

\section{Bacterial strains}

Two hundred and eighty MTB strains were respectively selected with a simple random sampling method from pulmonary TB patients seeking health care in Beijing Chest Hospital in 2005 and 2015. Of these 560 strains, 18 failed to grow upon subculture, and the remaining 542 were included for further analysis. All the bacterial cells were stored at $-80^{\circ} \mathrm{C}$ in Middlebrook 7H9 broth containing $10 \%$ glycerol. Prior to conducting drug susceptibility testing, the strains were recovered on Löwenstein-Jensen (L-J) medium for $4-6$ weeks at $37^{\circ} \mathrm{C}$. The demographic information and treatment history were obtained from the medical records.

\section{Conventional drug susceptibility testing and Mycobacterium species identification}

Conventional drug susceptibility testing was performed by the absolute concentration method as previously described [19]. This procedure was performed by inoculating L-J medium supplemented with INH or without drug with MTB. The concentration of INH in medium was $0.2 \mathrm{mg} / \mathrm{mL}$. In addition, the mycobacterium species identification was conducted by biochemical assays with medium containing p-nitrobenzoic acid (PNB) and 2thiophenecarboxylic acid hydrazide (TCH). Susceptibility to these compounds was defined as the growth of less than 20 colonies on the surface of L-J medium with the corresponding compounds.

\section{Minimal inhibitory concentration (MIC)}

To determine INH MICs of MTB strains, a microplate Alamar blue assay (MABA) was performed as described previously [20]. Briefly, the inoculum was prepared from the 4-week-old cultures grown in L-J medium. The turbidity of the cultures was adjusted to 1.0 McFarland standard. The final inoculum suspension was made by a 1:20 dilution in Middlebrook 7H9 broth containing 10\% OADC, and then $0.1 \mathrm{~mL}$ was delivered to the wells of the 96-well plate with serial two-fold dilutions of INH in $100 \mu \mathrm{L}$ of $7 \mathrm{H} 9$ broth. After 7 days of incubation, $70 \mu \mathrm{L}$ of Alamar Blue solution was added to each well, and incubated for another $24 \mathrm{~h}$ at $37^{\circ} \mathrm{C}$. A change from blue to pink indicates the bacterial growth. MIC was defined as the lowest concentration of INH that inhibited the color change from blue to pink. INH concentrations were doubling dilutions from 0.063 to $32 \mu \mathrm{g} / \mathrm{mL}$. The INH resistance was defined as an MIC of $\geq 0.5 \mu \mathrm{g} / \mathrm{mL}$, which was referred to the previous literature [21]. In addition, low-level resistance to INH was defined as an MIC of $\leq 2 \mu \mathrm{g} / \mathrm{mL}$; moderate-level resistance was defined as an MIC ranging from 4 to $16 \mu \mathrm{g} / \mathrm{mL}$; highlevel resistance was defined as an MIC of $\geq 32 \mathrm{mg} / \mathrm{L}$. M. tuberculosis H37Rv (ATCC 27249) was tested in both rounds as a quality control strain.

\section{DNA amplification and sequencing}

Crude genomic DNA was extracted from freshly cultured bacteria using the boiling lysis method as previously reported [19]. The partial fragments of $k a t G$ and the promoter region of inhA gene were amplified with the primers as listed in the Additional file 1: Table S1. The 
PCR mixture was prepared in a volume of $50 \mu \mathrm{L}$ containing $25 \mu \mathrm{L} 2 \times$ GoldStar Best MasterMix (CWBio, Beijing, China), $2 \mu \mathrm{L}$ of DNA template and $0.2 \mu \mathrm{M}$ of each primer set. The PCR products were sent to Qingke Company for DNA sequencing service. DNA sequences were aligned with the corresponding sequences of the reference $M$. tuberculosis $\mathrm{H} 37 \mathrm{Rv}$ strains using BioEdit software (http://www.mbio.ncsu.edu/bioedit/bioedit.html).

\section{Statistical analysis}

The rates of categorical variables between groups were compared using the Chi-square test (Fisher's exact test when the expected number was less than five). Statistical analysis was performed in SPSS 17.0 software (SPSS Inc., USA). Difference with $P<0.05$ was considered to indicate significant difference for chi-square test, while for the paired comparisons, the difference was considered as significant if $P$ value was less than false discovery rate (FDR) to reduce the false positive results [21].

\section{Results}

Factors associated with the increased prevalence of INHresistant TB

A total of 542 MTB isolates were enrolled in this study, including 273 isolates (50.4\%) collected in 2005 and 269 isolates $(49.6 \%)$ collected in 2015. The proportion of INH resistance $(39.0 \%, 105 / 269)$ in 2015 was significantly higher than in $2005(30.0 \%, 82 / 273 ; P=0.03)$. Similar, RIF resistance was identified in $36.4 \%(98 / 269)$ of MTB isolates in 2015, which was significantly higher than that in 2005 (28.2\%, $77 / 273, P=0.04)$. In addition, there was no significant difference in the proportion of MDR-TB between $2005(24.2 \%, 66 / 273)$ and $2015(31.2 \%, 84 / 269, P=0.07)$. We further analysed the distribution of INH-resistant TB with respect to sex, age and treatment history. As shown in Table 1, the proportion of male INH-resistant cases in 2015 $(65.7 \%, 69 / 105)$ was significantly higher than in 2005 (54.9\%, 45/82; $P=0.01$ ). Similarly, INH-resistance were found in 38 (22.5\%) of 169 new cases in 2005, with a significantly higher proportion of such cases observed in $2015(33.3 \%, 63 / 189 ; p=0.02)$. In contrast, the distributions of INH-resistant cases according to age had no statistically significant differences between 2005 and $2015(P>0.05)$.

\section{MICs and profiling of genetic mutations of INH-resistant MTB isolates}

The distribution of MTB isolates with different MIC values was summarized in Fig. 1. Overall, 67.8\% (185/273) and $53.2 \%$ (143/269) of MTB isolates tested had MIC values no more than $0.063 \mathrm{mg} / \mathrm{L}$ in 2005 and 2015, respectively. Among 269 isolates collected in 2015, 76 (28.3\%, 76/269) exhibited high-level INH-resistance (MIC $\geq 32 \mathrm{mg} / \mathrm{L}$ ), which was significantly higher than that in 2005 (20.5\%, 56/273, $P=0.04)$. We further investigated genetic mutation profiles among INH-resistant MTB isolates. As shown in Table 2, mutations within $k a t G$ and promoter of $i n h A$ were found in $82.9 \%(68 / 82)$ of INH-resistant isolates in 2005 and $81.9 \%(86 / 105)$ in 2015 , respectively. The katG mutation alone was observed most frequently, accounting for 56 in 2005 and 58 in 2015. In addition, inhA promoter region T$8 \mathrm{C}$ mutation alone was only identified in 4 isolates collected in 2015. Statistical analysis revealed a significantly higher percentage of INH-resistant isolates carrying inhA promoter mutations in 2015 (26.7\%) versus that in 2005 (14.6\%, $P=0.04$ ), while no significant difference was observed in the rates of isolates containing $\mathrm{kat} G$ mutations between 2005 (76.8\%) and 2015 (70.5\%, $P=0.33)$.

Table 1 Distribution of INH-resistant MTB isolates among demographic and clinical characteristics between 2005 and 2015 in this study

\begin{tabular}{|c|c|c|c|c|c|c|}
\hline \multirow[t]{3}{*}{ Characteristics } & \multicolumn{2}{|c|}{$2005(n=273)$} & \multicolumn{2}{|c|}{$\begin{array}{l}\text { No. }(\%) \text { of isolates } \\
2015(n=269)\end{array}$} & \multirow[t]{3}{*}{$P$ value } & \multirow[t]{3}{*}{ OR 95\% Cl } \\
\hline & \multirow{2}{*}{$\begin{array}{l}\text { INH-R } \\
(n=82)\end{array}$} & $\mathrm{INH}-\mathrm{S}$ & $\mathrm{INH}-\mathrm{R}$ & $\mathrm{INH}-\mathrm{S}$ & & \\
\hline & & $(n=191)$ & $(n=105)$ & $(n=164)$ & & \\
\hline \multicolumn{7}{|l|}{ Sex } \\
\hline Men & $45(54.9)$ & $123(64.4)$ & $69(65.7)$ & 103(62.8) & 0.01 & $1.83(1.16-2.89)$ \\
\hline Women & $37(45.1)$ & 68(35.6) & $36(34.3)$ & $61(37.2)$ & 0.78 & $1.08(0.61-1.93)$ \\
\hline \multicolumn{7}{|c|}{ Age group (years) } \\
\hline$<25$ & $9(11.0)$ & $22(11.5)$ & $9(8.6)$ & 19(11.6) & 0.80 & $1.16(0.38-3.51)$ \\
\hline $25-44$ & $34(41.5)$ & $72(37.7)$ & $42(40.0)$ & 54(32.9) & 0.09 & $1.65(0.93-2.92)$ \\
\hline $45-64$ & $28(34.1)$ & $64(33.5)$ & $39(37.1)$ & 59(36.0) & 0.18 & $1.51(0.83-2.76)$ \\
\hline$>64$ & $11(13.4)$ & $33(17.3)$ & 15(14.3) & $32(19.5)$ & 0.47 & $1.41(0.56-3.52)$ \\
\hline \multicolumn{7}{|c|}{ Treatment History } \\
\hline New case & $38(46.3)$ & 131(68.6) & $63(60.0)$ & $126(76.8)$ & 0.02 & $1.72(1.08-2.76)$ \\
\hline Re-treated & 44(53.7) & $60(31.4)$ & $42(40.0)$ & $38(23.2)$ & 0.17 & $1.51(0.84-2.71)$ \\
\hline
\end{tabular}




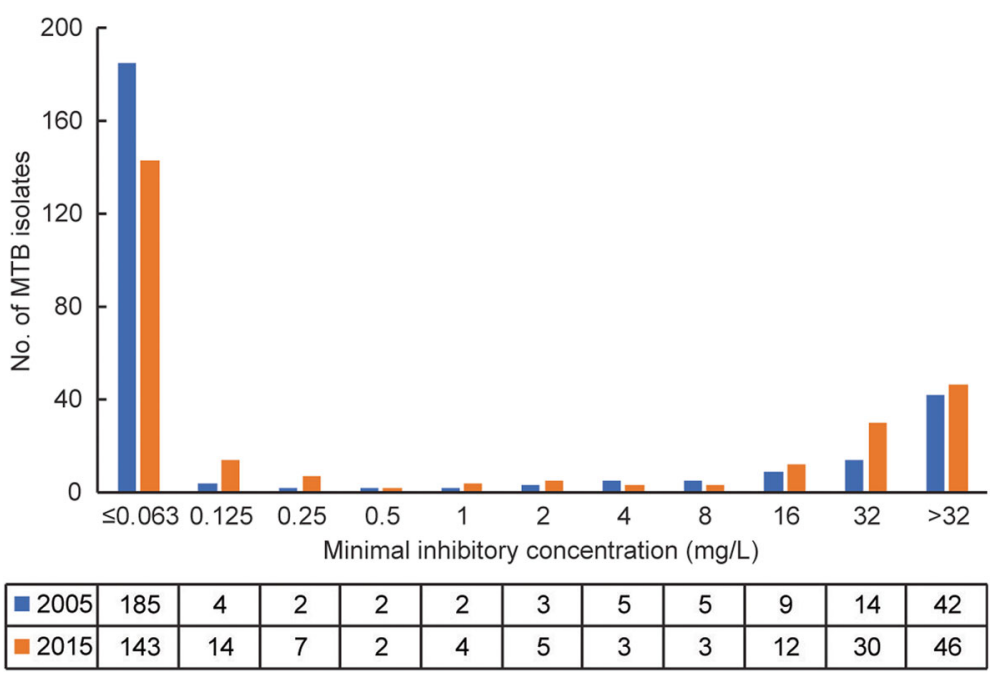

Fig. 1 Distributions of the INH MICs of the MTB isolates enrolled in this study

\section{Correlation between phenotypic resistant level and mutations conferring INH resistance}

We further compared the distribution and resistance levels to INH of MTB isolates harboring different mutation types. The results are summarized in Fig. 2. Generally, the highest proportion of MTB isolates with highlevel resistance was identified in those with mutations in both inhA and katG, which was significantly higher than that of isolates without mutation. Similarly, katG mutation alone correlated with high-level resistance and the moderate-level resistance, while was present significantly less often in the low-level resistant group compared to the group without mutation. In addition, there was no

Table 2 Comparison of mutant profiles in katG and inhA among INH-resistant isolates between 2005 and 2015

\begin{tabular}{llll}
\hline Mutation type & \multicolumn{2}{l}{$\begin{array}{l}\text { No. of INH-resistant isolates } \\
\text { with different mutations (\%) }\end{array}$} & \\
\cline { 2 - 3 } & $\begin{array}{l}2005 \\
(n=82)\end{array}$ & $\begin{array}{l}2015 \\
(n=105)\end{array}$ \\
\cline { 1 - 2 } $\begin{array}{lll}\text { Single Mutation } \\
\text { inhA-8 }\end{array}$ & $0(0.0)$ & $4(3.8)$ & 0.13 \\
inhA-15 & $5(6.1)$ & $8(7.6)$ & 0.69 \\
katG 315 & $56(68.3)$ & $58(54.3)$ & 0.07 \\
Total & $61(74.4)$ & $70(65.7)$ & 0.25 \\
Double Mutations & & & \\
inhA-8+katG 315 & $3(3.7)$ & $4(3.8)$ & 1.00 \\
inhA- 15+katG 315 & $4(4.9)$ & $12(11.4)$ & 0.11 \\
Total & $7(8.5)$ & $16(15.2)$ & 0.17 \\
With mutations & & & \\
inhA & $12(14.6)$ & $28(26.7)$ & 0.04 \\
katG & $63(76.8)$ & $74(70.5)$ & 0.33 \\
Without mutation & $14(17.1)$ & $19(18.1)$ & 0.86 \\
\hline
\end{tabular}

significant differences in the distribution and resistance levels to INH between group with inhA mutation alone and group without mutation $(P>0.05)$.

\section{Distribution of MTB isolates with inhA mutations}

We further analyzed the distribution of MTB isolates with inhA mutations according to patients' characteristics, so that to identify risk factors associated with the increased rate of MTB isolates harboring inhA promoter mutation in 2015. The results are summarized in Table 3. Notably, the proportion of MTB isolates with inhA mutations $(26.7 \%, 28 / 105)$ for patients who had previous exposure to protionamide (PTH) was higher than that for patients who had no previous exposure to PTH $(21.4 \%, 6 / 28)$. On the contrary, no significant different in prevalence was observed among patient sex, age and various drug exposure history groups $(P>0.05)$.

\section{Discussion}

Drug-resistant TB emerges as a major threat to effective TB control and the attainment of the 2035 global targets in China [22]. Our data demonstrate that the proportion of INH-resistant MTB isolates significantly increased during the last decade [19], which was mainly attributed to an increase of high-level INH-resistant MTB. Consistency with our results, the increasing prevalence of drug-resistant TB has been noted by several recent reports in China $[19,23]$. There is no doubt that the use of anti-TB drugs can affect the development of drug-resistant tubercle bacillus. Considering that INH is one of the most important first-line drug administered by TB patients during intensive and continuation phases, the prolonged exposure duration would facilitate the occurrence of INH-resistant bacteria. In addition, the fact that the choice of dose for antibiotic treatment can select various levels of drug 


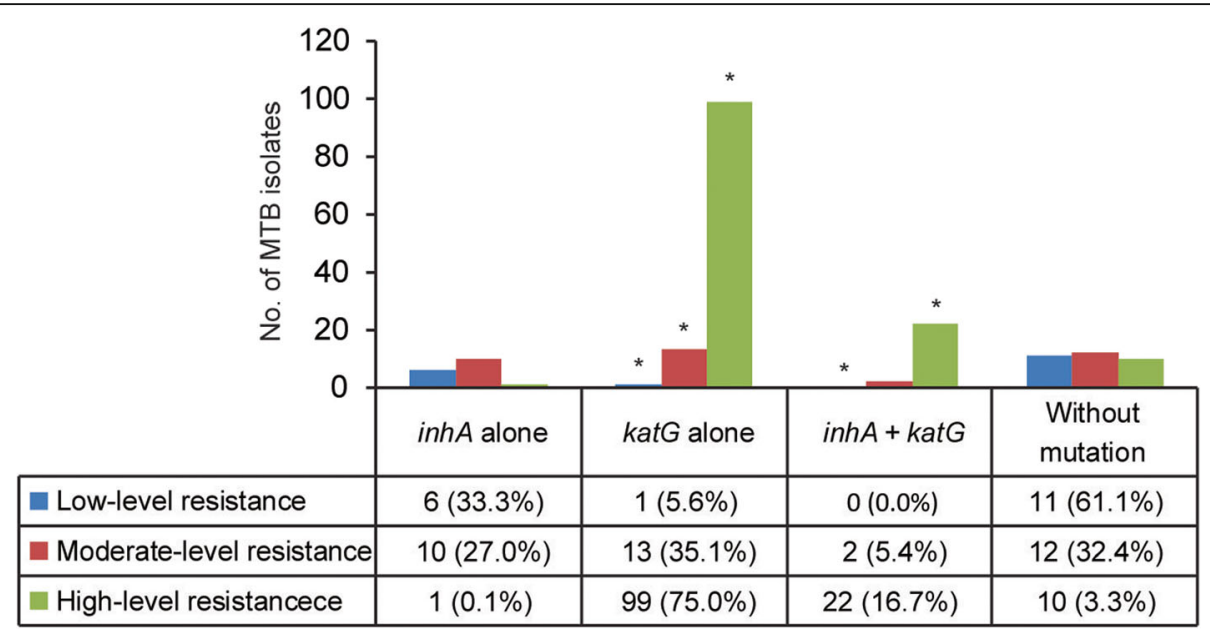

Fig. 2 Correlation between phenotypic resistant level and mutations conferring INH resistance. Low-level resistance: $0.5 \leq \mathrm{MIC} \leq 2 \mathrm{mg} / \mathrm{L}$; moderate-level resistance: $4 \leq \mathrm{MIC} \leq 16 \mathrm{mg} / \mathrm{L}$; high-level resistance: MIC $\geq 32 \mathrm{mg} / \mathrm{L}$; asterisk represents significant differences $[P$ value is less than the false discovery rate (FDR) 0.017 ] between the group with various mutation types and the group without mutations

resistance [24]. Although the dosage of INH for treatment of TB patients is not changed, the increasing prescription of p-aminosalicylic acid (PAS) [25], a competitive substrate that decreases in vivo acetylation and dehydrazination of INH, may elevate the INH concentration in patients receiving a combination of INH and PAS [26], thereby accelerating the emergence of high-level INH resistance.

Several recent reports have examined the contribution of kat $G$ and inhA promoter mutations in INH-resistant MTB isolates, and shown significant geographic diversity across regions [27]. In the present study, mutations in

Table 3 Factors associated with MTB strains harboring inhA mutation in 2015

\begin{tabular}{|c|c|c|c|c|}
\hline \multirow[t]{2}{*}{ Characteristics } & \multicolumn{2}{|c|}{ No. of INH-resistant isolates (\%) } & \multirow[t]{2}{*}{$P$ value } & \multirow[t]{2}{*}{ OR $(95 \% \mathrm{Cl})$} \\
\hline & $\begin{array}{l}\text { With inhA mutation } \\
(n=28)\end{array}$ & $\begin{array}{l}\text { Without inhA mutation } \\
(n=77)\end{array}$ & & \\
\hline \multicolumn{5}{|l|}{ Sex } \\
\hline Men & 18(64.3) & $51(66.2)$ & 0.85 & $1.09(0.44-2.70)$ \\
\hline Women & $10(35.7)$ & $26(33.8)$ & & 1.00(Ref) \\
\hline \multicolumn{5}{|c|}{ Age group (years) } \\
\hline$<25$ & $3(10.7)$ & $6(7.8)$ & 0.39 & $0.47(0.10-2.30)$ \\
\hline $25-44$ & $8(28.6)$ & $34(44.2)$ & & 1.00(Ref) \\
\hline $45-64$ & $12(42.9)$ & $27(35.1)$ & 0.22 & $0.53(0.19-1.48)$ \\
\hline$>64$ & $5(17.9)$ & $10(13.0)$ & 0.29 & $0.47(0.13-1.76)$ \\
\hline \multicolumn{5}{|c|}{ Treatment History } \\
\hline New case & $16(35.7)$ & $47(79.2)$ & & 1.00 (Ref) \\
\hline Re-treated & $12(64.3)$ & $30(20.8)$ & 0.72 & $0.85(0.35-2.05)$ \\
\hline \multicolumn{5}{|c|}{ Previous drug exposure } \\
\hline $\mathrm{INH}$ & $12(77.8)$ & $30(26.0)$ & 0.72 & $0.85(0.35-2.05)$ \\
\hline RIF & $12(77.8)$ & $30(26.0)$ & 0.72 & $0.85(0.35-2.05)$ \\
\hline EMB & 12(77.8) & $30(26.0)$ & 0.72 & $0.85(0.35-2.05)$ \\
\hline PZA & 12(77.8) & $30(26.0)$ & 0.72 & $0.85(0.35-2.05)$ \\
\hline SLID & $8(32.1)$ & 19(15.6) & 0.69 & $0.82(0.31-2.16)$ \\
\hline $\mathrm{FQ}$ & 10(39.3) & $22(20.8)$ & 0.48 & $0.72(0.29-1.80)$ \\
\hline PTH & 6(21.4) & $4(5.2)$ & 0.02 & $0.20(0.05-0.78)$ \\
\hline
\end{tabular}


inh $A$ alone were observed in $11.4 \%$ resistant isolates in 2015. The frequency of such mutants conferring INH resistance is similar to that in Chongqing (10\%) [28] and that in China (11\%) [16], although it is lower than that in Philippines (22\%) [29] and that in South Africa (30\%) [30], and higher than that in Lithuania (3.4\%) [5] and that in Poland (4\%) [31]. The regional differences in the frequencies of mutations associated with INH resistance may reflect the diversity in molecular characteristics of predominant MTB isolates circulating in geographically distinct areas, and also provide hints for the development of molecular-based diagnostic tests.

In line with previous studies, in vitro experimental data of this study indicated that katG codon 315 mutations are associated with high-level resistance to INH, while mutations in inhA regulatory region confer lowlevel resistance to INH [12]. We also observed that 3.3\% of INH-resistant isolates without $k a t G$ and inhA mutation exhibited high-level resistance. Generally, efflux pump and other natural mechanisms always medicate low-level resistance, while the high-level resistance is due to mutations in the target genes [3]. Hence, we speculate that mutations in other candidates, such as oxyR-ahpC intergenic region, kasA, and $n d h$, may be responsible for these isolates exhibiting high-level resistance [11]. Further experimental data will extend our knowledge on molecular mechanisms of novel genes involving INH resistance in MTB.

Another interesting finding of our report was that the prevalence of INH-resistant isolates carrying mutations in inhA regulatory region was significantly increased between 2005 and 2015. In addition, our analysis results showed a significant association between inhA promoter mutations and previous PTH exposure. A serial of previous studies has reported that inhA promoter mutations not only cause INH resistance, but they also confer cross-resistance to ethionamide (ETH) and PTH [32]. In most cases, PTH is used as a subsequent choice for the patients suffering treatment failure with the INH-containing first-line regimen. More importantly, a recent survey indicates that there was a significant increasing trend for PTH prescribing for TB patients in China during the past years [33]. Hence, the preexisting inhA promoter mutations may facilitate successful adaptation to the PTH exposure at low fitness cost for INH-resistant MTB isolates. Despite strong evidence linking inhA promoter mutations and ETH/PTH resistance for MTB, these mutations may play a compensatory role in increasing their fitness due to the absence of experimental data in the present study. Therefore, further in vitro experiments are urgently needed to clarify the selective advantage of these mutants during exposure to ETH/PTH.

Our study has several important hints for clinical use of INH in the treatment of TB patients. On one hand, high-dose INH has been included in the WHO guidelines for the treatment of MDR patients with low-level INH resistance [34]. However, the increasing prevalence of high-level INH resistance among MTB isolates may impair the effectiveness of high-dose INH for these patients in China. On the other hand, considering that $i n h A$ promoter mutations generate additional cross-resistance to PTH. The inclusion of PTH in the regimen should be used for the treatment of INH-resistant patients without $i n h A$ promoter mutation. Taken together, these data underscore the need to clarify the specific resistance mutations for INHresistant TB cases in order to guide clinicians in making therapeutic decisions appropriately.

\section{Conclusion}

In conclusion, our results demonstrated that the proportion of INH-resistant MTB isolates significantly increased during the last decade, which was mainly attributed to an increase of high-level INH-resistant MTB. In addition, prior exposure to PTH may be associated with the increased frequency of INH-resistant tuberculosis strains with inhA mutations in China. Therefore, our findings highlight the need to clarify the specific resistance mutations for INH-resistant TB cases in order to guide clinicians in making therapeutic decisions appropriately.

\section{Additional file}

Additional file 1: Table S1. Primers used for DNA sequencing. (DOCX $15 \mathrm{~kb}$ )

\section{Abbreviations}

ETH: ethionamide; FDR: false discovery rate; INH: Isoniazid; L-J: LowensteinJensen; MABA: microplate Alamar blue assay; MDR-TB: multidrug-resistant TB; MIC: minimal inhibitory concentration; MTB: Mycobacterium tuberculosis; PAS: para-aminosalicylic acid; PNB: p-nitrobenzoic acid; PTH: protionamide; RIF: rifampicin; TB: tuberculosis; TCH: 2-thiophenecarboxylic acid hydrazide

\section{Acknowledgements}

We would like to thank the staffs from National Clinical Laboratory on Tuberculosis for their technical assistance.

\section{Authors' contributions}

Conceived and designed the experiments: FH, JL, YP, HH. Performed the experiments: FH, JL, ZZ, WJ, JS, YM, YP. Analyzed the data: JL, LD, LZ, YW, YP. Wrote the paper: FH, ZZ, YP, HH. All authors contributed to the final manuscript. All authors read and approved the final manuscript.

\section{Funding}

This study was supported by the Beijing Municipal Administration of Hospitals' Youth Programme (QML20171601), Tongzhou District Science and Technology Committee (KJ2017CX076), Beijing Bio-Bank of Clinical Resources on Tuberculosis (D131100005313012), Beijing Municipal Administration of Hospitals' Ascent Plan (DFL20181602), Beijing Municipal Administration of Hospitals Clinical Medicine Development of Special Funding Support (ZYLX201809). The funders had no role in study design, data collection and analysis, decision to publish, and preparation of the manuscript.

Availability of data and materials

The datasets used and/or analysed during the current study available from the corresponding author on reasonable request. 


\section{Ethics approval and consent to participate}

The protocols applied in this study were approved by the Ethics Committee of the Beijing Chest Hospital, Capital Medical University. Patient records/ information was anonymized and de-identified prior to analysis, and informed consent was not obtained from each participant.

\section{Consent for publication}

Not applicable.

\section{Competing interests}

The authors declare that they have no competing interests.

\section{Author details}

'National Clinical Laboratory on Tuberculosis, Beijing Key laboratory on Drug-resistant Tuberculosis Research, Beijing Chest Hospital, Capital Medical University, Beijing Tuberculosis and Thoracic Tumor Institute, No. 97, Machang, Tongzhou District, Beijing 101149, China. ${ }^{2}$ Beijing Key Laboratory for Pediatric Diseases of Otolaryngology, Head and Neck Surgery, Beijing Pediatric Research Institute, Beijing Children's Hospital, Capital Medical University, National Center for Children's Health, Beijing, China.

\section{Received: 4 September 2018 Accepted: 29 July 2019}

\section{Published online: 05 August 2019}

\section{References}

1. World Health Organization (WHO). Global tuberculosis report 2018. Geneva: WHO; 2018. WHO/CDS/TB/2018.20

2. Abubakar I, Zignol M, Falzon D, Raviglione M, Ditiu L, Masham S, Adetifa I, Ford N, Cox H, Lawn SD, et al. Drug-resistant tuberculosis: time for visionary political leadership. Lancet Infect Dis. 2013;13(6):529-39.

3. Zhang Z, Pang Y, Wang Y, Liu C, Zhao Y. Beijing genotype of Mycobacterium tuberculosis is significantly associated with linezolid resistance in multidrug-resistant and extensively drug-resistant tuberculosis in China. Int J Antimicrob Agents. 2014;43(3):231-5.

4. Gandhi NR, Nunn P, Dheda K, Schaaf HS, Zignol M, van Soolingen D, Jensen P. Bayona J. Multidrug-resistant and extensively drug-resistant tuberculosis: a threat to global control of tuberculosis. Lancet. 2010;375(9728):1830-43.

5. Bakonyte D, Baranauskaite A, Cicenaite J, Sosnovskaja A, Stakenas P. Molecular characterization of isoniazid-resistant Mycobacterium tuberculosis clinical isolates in Lithuania. Antimicrob Agents Chemother. 2003;47(6): 2009-11.

6. Jacobson KR, Theron D, Victor TC, Streicher EM, Warren RM, Murray MB. Treatment outcomes of isoniazid-resistant tuberculosis patients, Western Cape Province, South Africa. Clin Infect Dis. 2011;53(4):369-72.

7. Cattamanchi A, Dantes RB, Metcalfe JZ, Jarlsberg LG, Grinsdale J, Kawamura LM, Osmond D, Hopewell PC, Nahid P. Clinical characteristics and treatment outcomes of patients with isoniazid-monoresistant tuberculosis. Clin Infect Dis. 2009;48(2):179-85.

8. Van Deun A, Martin A, Palomino JC. Diagnosis of drug-resistant tuberculosis: reliability and rapidity of detection. Int J Tuberc Lung Dis. 2010;14(2):131-40.

9. Zhang Y, Heym B, Allen B, Young D, Cole S. The catalase-peroxidase gene and isoniazid resistance of Mycobacterium tuberculosis. Nature. 1992; 358(6387):591-3.

10. Sherman DR, Mdluli K, Hickey MJ, Arain TM, Morris SL, Barry CE 3rd, Stover CK. Compensatory ahpC gene expression in isoniazid-resistant Mycobacterium tuberculosis. Science. 1996;272(5268):1641-3.

11. Zhang $Y$, Yew WW. Mechanisms of drug resistance in Mycobacterium tuberculosis: update 2015. Int J Tuberc Lung D. 2015;19(11):1276-89.

12. Vilcheze $C$, Jacobs WR. The mechanism of isoniazid killing: clarity through the scope of genetics. Annu Rev Microbiol. 2007:61:35-50.

13. Silva MSN, Senna SG, Ribeiro MO, Valim ARM, Telles MA, Kritski A, Morlock GP, Cooksey RC, Zaha A, Rossetti MLR. Mutations in katG, inhA and ahpC genes of Brazilian isoniazid-resistant isolates of Mycobacterium tuberculosis. J Clin Microbiol. 2003;41(9):4471-4.

14. Guo HL, Seet Q, Denkin S, Parsons L, Zhang Y. Molecular characterization of isoniazid-resistant clinical isolates of Mycobacterium tuberculosis from the USA. J Med Microbiol. 2006;55(11):1527-31.

15. Zhao YL, Xu SF, Wang LX, Chin DP, Wang SF, Jiang GL, Xia H, Zhou Y, Li Q Ou XC, et al. National Survey of drug-resistant tuberculosis in China. New Engl J Med. 2012;366(23):2161-70.
16. Zhang ZJ, Lu J, Liu M, Wang YF, Qu GP, Li HX, Wang JC, Pang Y, Liu CT, Zhao YL. Genotyping and molecular characteristics of multidrug-resistant Mycobacterium tuberculosis isolates from China. J Inf Secur. 2015;70(4):335-45.

17. Yao C, Zhu T, Li Y, Zhang L, Zhang B, Huang J, Fu W. Detection of rpoB, katG and inhA gene mutations in Mycobacterium tuberculosis clinical isolates from Chongqing as determined by microarray. Clin Microbiol Infec. 2010;16(11):1639-43

18. Chen QY, Pang Y, Liang QF, Lin SF, Wang YF, Lin J, Zhao Y, Wei SZ, Zheng JF, Zheng SH. Molecular characteristics of MDR Mycobacterium tuberculosis strains isolated in Fujian, China. Tuberculosis. 2014;94(2):159-61.

19. Huo F, Luo J, Shi J, Zong Z, Jing W, Dong W, Dong L, Ma Y, Liang Q, Shang $Y$, et al. Increasing prevalence of rifampicin-resistant Mycobacterium tuberculosis is associated with the transmission of strains harboring compensatory mutations in China: a 10-year comparative analysis. Antimicrob Agents Chemother. 2018

20. Zhang Z, Li T, Qu G, Pang Y, Zhao Y. In vitro synergistic activity of clofazimine and other antituberculous drugs against multidrug-resistant Mycobacterium tuberculosis isolates. Int J Antimicrob Agents. 2015;45(1):71-5.

21. Jing $W$, Pang $Y$, Zong Z, Wang J, Guo R, Huo F, Jiang G, Ma Y, Huang $H$, Chu N. Rifabutin resistance associated with double mutations in rpoB gene in Mycobacterium tuberculosis isolates. Front Microbiol. 2017;8:1768.

22. Mariandyshev A, Eliseev P. Drug-resistant tuberculosis threatens WHO's endTB strategy. Lancet Infect Dis. 2017;17(7):674-5.

23. Pang $Y$, Zhang Z, Wang Y, Wang S, Song Y, Zhao B, Zhou Y, Ou X, Li Q, Xia $\mathrm{H}$, et al. Genotyping and prevalence of pyrazinamide- and moxifloxacinresistant tuberculosis in China, 2000 to 2010. Antimicrob Agents Chemother 2017:61:2.

24. Olofsson SK, Cars O. Optimizing drug exposure to minimize selection of antibiotic resistance. Clin Infect Dis. 2007;45(Suppl 2):S129-36.

25. An J, Bai X, Gao M, Chu N, Huang H, Xu H, Zhang X, Li B, Huang C, Cai H, et al. Anti-tuberculosis drug prescribing for inpatients in a National Tuberculosis Hospital of China, 2011-2015. J Glob Antimicrob Resist. 2018.

26. Devadatta S, Bhatia AL, Andrews RH, Fox W, Mitchison DA, Radhakrishna S, Ramakrishnan CV, Selkon JB, Velu S. Response of patients infected with isoniazid-resistant tubercle bacilli to treatment with isoniazid plus PAS or isoniazid alone. Bull World Health Organ. 1961;25:807-29.

27. Seifert M, Catanzaro D, Catanzaro A, Rodwell TC. Genetic mutations associated with isoniazid resistance in Mycobacterium tuberculosis: a systematic review. PLoS One. 2015;10(3):e0119628.

28. Zhang D, Liu B, Wang Y, Pang Y. Rapid molecular screening for multidrugresistant tuberculosis in a resource-limited region of China. Tropical Med Int Health. 2014;19(10):1259-66.

29. Herrera L, Valverde A, Saiz P, Saez-Nieto JA, Portero JL, Jimenez MS. Molecular characterization of isoniazid-resistant Mycobacterium tuberculosis clinical strains isolated in the Philippines. Int J Antimicrob Agents. 2004; 23(6):572-6.

30. Muller B, Streicher EM, Hoek KGP, Tait M, Trollip A, Bosman ME, Coetzee GJ, Chabula-Nxiweni EM, Hoosain E, Gey van Pittius NC, et al. inhA promoter mutations: a gateway to extensively drug-resistant tuberculosis in South Africa? Int J Tuberc Lung D. 2011;15(3):344-51.

31. Jagielski T, Bakula Z, Roeske K, Kaminski M, Napiorkowska A, AugustynowiczKopec E, Zwolska Z, Bielecki J. Detection of mutations associated with isoniazid resistance in multidrug-resistant Mycobacterium tuberculosis clinical isolates. J Antimicrob Chemother. 2014;69(9):2369-75.

32. Tan $Y$, Su B, Zheng $H$, Song $Y$, Wang $Y$, Pang Y. Molecular characterization of Prothionamide-resistant Mycobacterium tuberculosis isolates in southern China. Front Microbiol. 2017;8:2358.

33. An J, Bai X, Gao M, Chu N, Huang H, Xu H, Zhang X, Li B, Huang C, Cai $\mathrm{H}$, et al. Antituberculosis drug prescribing for inpatients in a national tuberculosis hospital in China, 2011-2015. J Glob Antimicrob Resist. 2018:14:17-22

34. Falzon D, Schunemann HJ, Harausz E, Gonzalez-Angulo L, Lienhardt C, Jaramillo E, Weyer K. World Health Organization treatment guidelines for drug-resistant tuberculosis, 2016 update. Eur Respir J. 2017;49:3.

\section{Publisher's Note}

Springer Nature remains neutral with regard to jurisdictional claims in published maps and institutional affiliations. 